\title{
Identification of transcriptional pathways in naturally occurring human regulatory $\mathrm{T}$ cells

\author{
A Tuettenberg*1, T Fischer ${ }^{2}$, S Fondel ${ }^{1}$, B Markova ${ }^{2}$, J Kubach ${ }^{1}$, C Becker ${ }^{1}$, \\ K Satoh $^{1}$, J Joore ${ }^{3}$, S Stoll ${ }^{1}$ and H Jonuleit ${ }^{1}$
}

\begin{abstract}
Address: ${ }^{1}$ Department of Dermatology, Johannes Gutenberg-University, Mainz, Germany, ${ }^{2}$ Department of Hematology, Johannes GutenbergUniversity, Mainz, Germany and 3Pepscan Presto B.V., Array Technologies, Lelystad, The Netherlands

* Corresponding author
\end{abstract}

from 12th Joint Meeting of the Signal Transduction Society (STS). Signal Transduction: Receptors, Mediators and Genes

Weimar, Germany. 29-3I October 2008

Published: 26 February 2009

Cell Communication and Signaling 2009, 7(SuppI I):A9 doi:10.1186/1478-8IIX-7-SI-A9

This abstract is available from: http://www.biosignaling.com/content/7/SI/A9

(C) 2009 Tuettenberg et al; licensee BioMed Central Ltd.

Naturally occurring CD4+CD25 Tregs (nTregs) are essential for $\mathrm{T}$ cell homeostasis and the maintenance of peripheral tolerance. They prevent the activation of autoaggressive $\mathrm{T}$ cells in the context of autoimmune diseases and suppress inadequate allergen specific T cells. On the opposite, nTregs inhibit also effective immune responses against tumors such as melanoma. A detailed understanding of molecular mechanisms that control the functional properties of human nTregs is mandatory for the development of novel immunotherapies against allergy, autoimmunity and cancer. Therefore, we initiated a genomic, proteomic and kinome profiling of human nTregs to identify key molecules in human nTregs associated with their functional activation which are responsible for their state of anergy and/or their suppressive activity. We started with large-scale isolation of nTregs using whole leukapheresis products followed by polyclonal stimulation and analysis at different time points. As a result, we identified a distinct molecular activation pattern specific for the activation state of human nTregs. The impact of identified key molecules was tested in functional assays using specific inhibitors and siRNA mediated knockdown of these targets. A general transcriptional network analysis is currently under investigation and will be presented on the meeting. The main objective of our analysis is the identification of novel targets for the immunotherapeutic intervention of dysregulated immune responses in the near future. 